\title{
Influence of Biometry on Modern Intraocular Lens Surgery
}

\author{
a report by \\ Gerd U Auffarth
}

Chief of Faculty, and Vice Chairman, Department of Ophthalmology, University of Heidelberg

DOI: $10.17925 / E O R .2009 .02 .01 .30$

Cataract surgery with intraocular lens (IOL) implantation has become the most frequent type of surgical intervention in medicine. The success of this treatment is derived from several factors, such as surgical techniques, surgical tools, implant designs and peri-operative diagnostics.

As high standards have been reached in cataract surgery, IOL implantation has become a safe and reliable procedure. However, IOL calculation was still somewhat difficult when ultrasound measurement alone was the gold standard.

After the introduction of partial coherence interferometry $(\mathrm{PCI})$ in the shape of the IOL-Master (Zeiss), there were rapid developments in axial length measurement and, consequently, IOL calculation, and the potential for mismeasurement of total axial length suddenly became negligible. Individualisation and optimisation of A-constants made IOL calculation and calculation formulae more accurate. New optical biometers such as the LENSTAR LS 900 (Haag-Streit) and the ALLEGRO Biograph (WaveLight), which provide the surgeon with more data on factors such as lens thickness, anatomical anterior chamber depth and retinal thickness, will probably promote the further development of new IOL power formulae.

For special IOLs such as multifocal (MIOLs), toric or accommodative IOLs, optimal IOL power calculation is extremely important. In the 1990s, one of the biggest arguments against MIOLs was the insufficient accuracy of MIOL calculation; this argument is no longer used due to the improvements discussed above. Most optical biometers measure axial length as well as K-values of the cornea. These data are therefore crucial for the special IOLs. For toric IOLs, surgeons can also rely on the accurate data provided by laser interferometric biometers. As patients often have to pay for these special implants themselves, they too demand a high level of accuracy. Cataract surgery is now evolving into a refractive procedure. Refractive lens exchange is becoming more and more popular. For high hyperopic or myopic patients, refractive lens exchange has become the only choice. In presbyopia treatment, refractive lens exchange with MIOL implantation is becoming increasingly common. Toric implants are now widely used.

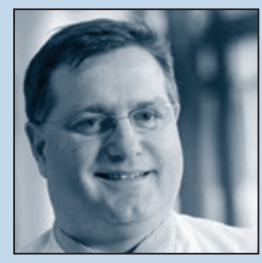

Gerd U Auffarth is Chief of Faculty and Vice Chairman of the Department of Ophthalmology at University of Heidelberg. He is a Board Member and Secretary of the German Society for Cataract and Refractive Surgery, Head of the International Vision Correction Research Centre (IVCRC) and a board member of the European Society of Cararact and Refractive Surgery (ESCRS)

E: auffarthg@aol.com
Figure 1: New Optical Biometers Provide More Data Along the Visual Optical Line

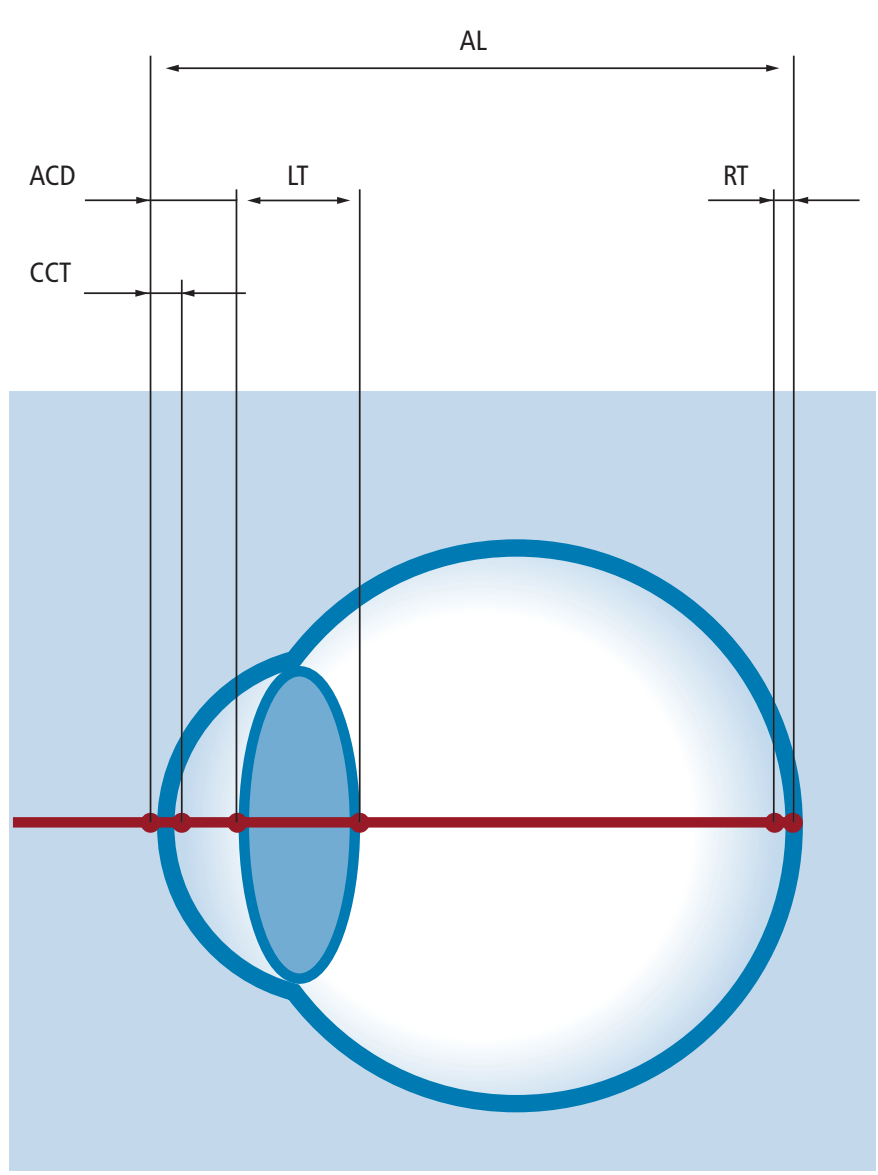

$A C D=$ anterior chamber depth (including $C C T$, anatomical without $C C T$ ); $C C T$ = central corneal thickness; $A L=$ axial length; $L T=$ lens thickness; $R T=$ retinal thickness.

The trend today is towards an individually customised approach in terms of IOL selection and manufacturing. New developments such as the Calhoun light-adjustable IOL underline this move towards personalisation of treatment, and in the long term could even allow wavefront-guided corrections to the IOL.

In summary, the introduction of optical biometry led to axial length measurement and IOL calculation becoming so reliable that presbyopic lens exchange, use of special IOLs (MIOLs, toric IOLs) and individualisation of patient treatment options are now possible. The combination of improved diagnostic tools and developments in IOL technology has shifted lens-based surgery from curative cataract procedures to functional refractive interventions.

The author has no financial interest in the products mentioned here. 


\section{The Centre Cannot Hold - New Visual Orientation Functions Found in the Brain}

Neurons in the superior colliculus (SC) - a paired structure below the thalamus that surrounds the pineal gland - are responsible for eye movements and eye-head co-ordination towards and away from visual stimuli. However, they may also perform a 'supervisory function' in analysing complex movement, according to a study at the Systems Neurobiology Laboratory of the Salk Institute in La Jolla. Researchers found that the SC may actually be responsible for inferring location information about moving objects, for instance helping the brain to identify the centre of an aeroplane when seen at night, when only the strobe lighting on the wing is visually detectable.

Lead researchers Ziad Hafed, PhD, and Richard Krauzlis, PhD, designed experiments wherein subjects had to infer the invisible centre of a visual target consisting only of two peripheral features and track its movement for several seconds. Interestingly, the central neurons were active during this tracking behaviour even without a visual stimulus in the centre of gaze.

In a second study, jointly with Laurent Goffart, PhD, a Professor at the Institut de Neurosciences Cognitives de la Méditerranée in Marseille, researchers inactivated a subset of SC neurons. They found that a subject's ability to track objects shifted away from the invisible centre, further suggesting that the $\mathrm{SC}$ is important in defining object location and centre. "By showing that the SC is not just a motor map, but also a map of behaviourally relevant object locations, our results provide a conceptual framework for understanding the role of the SC in non-motor functions such as visual attention and the functional links between motor control and sensory processing," says Hafed.

\section{Superior Colliculus}

The SC (from the Latin 'higher hills') and inferior colliculus (from the Latin 'lower hills') conjoin within the brain's tectal area. As a unit, they are thought to help orientate the head and eyes towards visual and audio stimuli.

\section{Related Papers}

Bergeron A, et al., Superior colliculus encodes distance to target, not saccade amplitude, in multi-step gaze shifts, Nat Neurosci, 2003;6(4):332-3.

Munoz DP, et al., Movement of neural activity on the superior colliculus motor map during gaze shifts, Science, 1991;251(4999): 1358-60.

Krauzlis $R$, et al., Target selection and the superior colliculus: goals, choices and hypotheses, Vision Research, 2004;44(12):1445-51.

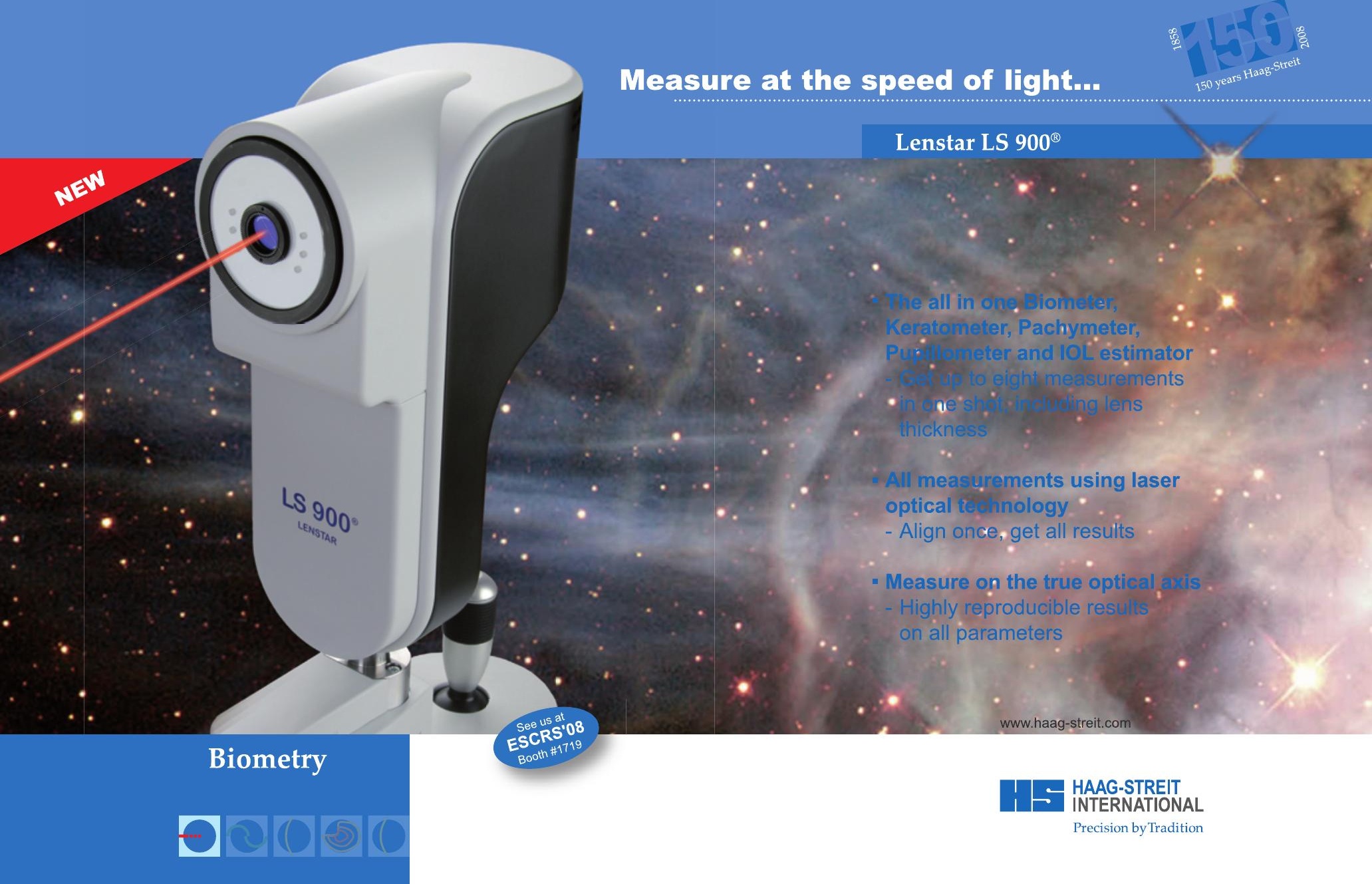

